\title{
Twin study - genetic comparison of matrix versus intramatrix rotation in the mandible and three different occlusal planes
}

\author{
Jin Hyeong Kim ${ }^{1+}$, Young Ho Kim', Soo Jin Kim¹, Joohon Sung ${ }^{1}$, Yun-Mi Song ${ }^{3}$, Jeong Won Shin ${ }^{1}$,
} Jae Hyun Park ${ }^{4}$ and Hwa Sung Chae ${ }^{1 *}$ (D)

\begin{abstract}
Background: The purpose of this study is to investigate the heritability of total rotation, matrix rotation, and intramatrix rotation of the mandible in Korean monozygotic (MZ) twins, dizygotic (DZ) twins, and their siblings.

Materials and methods: The samples consisted of 75 pairs of Korean twins (39.7 + 9.26 years; MZ group, 36 pairs; DZ group, 13 pairs; sibling group, 26 pairs). Lateral cephalograms were taken, and 13 variables related to internal and external mandible rotation were measured. Three types of occlusal planes (bisected occlusal plane, functional occlusal plane, and the MM bisector occlusal plane) were used to evaluate genetic influence on the occlusal plane. Heritability $\left(h^{2}\right)$ was calculated by using the intraclass correlation coefficient (ICC) and Falconer's method.

Results: With regard to mandibular rotation, the MZ twin group showed significantly higher ICC values compared to the DZ twin and sibling groups. The ICC mean values for 13 cephalometric measurements were 0.85 (MZ), 0.62 (DZ), and 0.52 (siblings) respectively. The heritability of the total rotation (0.48) and matrix rotation (0.5) between the $M Z$ and DZ groups was higher than that of the intramatrix rotation $(-0.14)$. All of the three types of occlusal plane showed high heritability, and among the three types, the functional occlusal plane showed the highest heritability $\left(h^{2}=0.76\right)$.

Conclusion: Based on these findings that showed a strong genetic effect on total rotation and matrix rotation, maintaining these rotations should be carefully considered in the orthodontic treatment plan, while the lower border of the mandible may be responsive to various treatments. Occlusal plane change, especially with regard to the functional occlusal plane, may not be stable due to strong genetic influences.
\end{abstract}

Keywords: Twins, Heritability, Mandibular rotation, Occlusal plane

\section{Background}

Evaluation of bone growth patterns is one of the most important factors in establishing treatment plans for growing patients. However, it was not until the middle of the 1900s that research on these patterns was thoroughly performed despite their importance. This was because of the complex properties of the bone growth

\footnotetext{
* Correspondence: hwasungchae@gmail.com

${ }^{\dagger}$ Jin Hyeong Kim and Young Ho Kim contributed equally to this work. 'Department of Orthodontics, Institute of Oral Health Science, Ajou University School of Medicine, Suwon, South Korea

Full list of author information is available at the end of the article
}

process in which condylar growth and remodeling of the mandible take place at the same time.

So far, studies have been concentrated on locating bone growth centers or evaluating the range of rotation by location. Furthermore, there have been attempts to visualize matrix rotation and intramatrix rotation within the jaw. A metallic implant study has enabled the ability to distinguish condylar and bone remodeling growth, and the concept of rotational growth was introduced as the bony core [1].

Björk and Skieller distinguished mandibular growth rotation into total rotation, matrix rotation, and intramatrix 
rotation using implant superimposition method. Björk and Skieller called the implant line inclination change as the "total rotation." This refers to the angle between the mandibular core and the SN plane, which shows the rotation of the core of the mandible "matrix rotation" is the combined transversions of the condyle growth between the skull base and the core of the mandible and the modification of the mandible due to bone remodeling. The measurements are a clinical interpretation that represents the mandible position and change of inclination. After overlapping two cephalometric x-rays, Björk and Skieller found a difference in the implant inclination and mandible inclination relative to the SN plane. They called this the "intramatrix rotation," and it signifies the independent rotation of the mandibular core that appears inside the soft tissue matrix [2].

Proffit et al. described Björk's total rotation as internal rotation, which is masked by surface changes and alterations in the rate of tooth eruption. Proffit et al. also explained that surface changes produce external rotation that is compensated for by internal rotation [3]. To prevent confusion among terms, the present study follows Björk's definition of rotation. Ricketts corpus axis (Xi-Pm) replaces Björk's core of mandible. The other two planes are the same.

Despite studies that investigated the mechanisms and contributions of each rotation in the total mandibular rotation, there has been no study on heritability of the rotation within the mandible.

Genetic factors that affect human craniofacial structures were identified through an experiment that used monozygotic twins (MZ), dizygotic twins (DZ), and their siblings [4]. The effect of heredity on some measurements was studied using the comparison among MZ, DZ, and sibling groups, which was efficient and useful in analyzing the heritability of measurements of interest [5].

To date, there have been many twin studies of the craniofacial area, most of which were performed using lateral cephalograms from $\mathrm{MZ}, \mathrm{DZ}$, and sibling groups and the predicted heritability of cephalometric parameters. Johannsdottir et al. stated that cephalometric data could support predictions when detecting genetic variations that affect complex polygenetic multifactorial traits [6].

In 1965, Hunter reported that the height dimension showed higher heritability than measures of facial depth related to dentition when using lateral cephalometric radiographs obtained from 72 pairs of like-sexed twins [7].

Manfredi analyzed 39 lateral cephalometric parameters and reported different inheritance trends. The highest concordance of values was seen between MZ pairs when compared with DZ pairs of the same sex singleton paired group. He also showed high heritability in the craniofacial region. Heritability seems to be expressed more anteriorly than posteriorly. Mandibular shape seems to be more genetically determined than mandibular size [8].
Carels et al. analyzed 23 hard tissue variables and found that genetic determination was higher in vertical than horizontal measurements and found a higher genetic component for boys in anterior facial height than for girls [9].

In 2004, Naini and Moss also proposed that lower anterior parts of the face were under strong genetic control [10]. Amini et al. found higher heritability in vertical variables compared to horizontal ones. The authors also suggested that heritability seemed to be expressed more anteriorly than posteriorly [11].

A recent study published in the European Journal of Orthodontics, 2016, analyzed 39 cephalometric variables in 141 pairs of twins. The results were in agreement with that of the Manfredi study that found the shape and sagittal position of the mandible were under stronger genetic control than its size and vertical relationship to the cranial base. The authors also reported that the polygon of the face-similarity was under strong genetic control and this might explain the profile resemblance between twins [12].

Lobb [13] reported high heritability in the angle between the occlusal and mandibular planes. The occlusal plane was distinguished as three, bisected occlusal planes by Downs [14], the functional occlusal plane by Wits [15], and as an MM (maxillary-mandibular) bisector by Hall-Scott [16].

The purpose of this study was to investigate which rotation has stronger heritability, between the matrix and intramatrix rotations of the mandible using lateral cephalometric measurements in Korean twins, to investigate its relevance to clinical orthodontic treatments, to compare each occlusal plane, and to investigate which occlusal plane was under the strongest hereditary influence.

\section{Materials and methods Study sample}

Among 553 Korean patients who participated in twin studies conducted at Samsung Medical Center from July 2011 to February 2012, a total of 75 pairs of twins whose hard tissue cephalometric measurements were available were included in this study: $36 \mathrm{MZ}$ twins (males, 16 pairs; females, 20 pairs), $13 \mathrm{DZ}$ twins (males, 7 pairs; females, 6 pairs), and 26 same sex sibling pairs (males, 11 pairs, females, 15 pairs). This study used the questionnaire of zygosity diagnosis (QOZD) method developed by Song et al. [17], which was proved to be effective to identify the types of twins.

Those who had undergone orthodontic treatment, orthognathic surgery such as two jaw surgery, those who had an edentulous area within the anterior teeth that could affect facial profile, and those who had a removable prosthesis which could affect the vertical dimension of the face were excluded from this study. 
The mean age of the subjects was 39.7 years old, and all the DZ twins and siblings were the same sex. In order to minimize the age influence, sibling pairs were selected with an age difference of less than 5 years. The demographic data explaining the study sample is shown in Table 1.

This study was approved by our Institutional Review Board (IRB, IRB 2005-08-113-027) and informed consent was signed by all subjects.

\section{Cephalometric measurements}

Lateral cephalograms were taken in the natural head position, and all measurements were analyzed by one researcher $(\mathrm{Kim} \mathrm{JH})$ using the $\mathrm{V}$-ceph 7.0 digital program (Cybermed, Seoul, South Korea). To verify measurement error, repeated tracings and measurements were performed at a 2-week interval on ten randomly selected patients. Measurement error was estimated for two sets of data using Dahlberg's formula [18].

Landmarks, reference lines, and cephalometric measurements are illustrated in Figs. 1, 2, and 3. To measure matrix rotation, intramatrix rotation, and variables related to occlusal planes, 13 variables were selected based on Rickett's analysis, Wits' analysis, Downs' analysis, and Hall-Scott's analysis. Among the three planes defined by Björk, we used the $\mathrm{SN}$ and the mandibular plane. The only difference was the mandibular core. The mandibular core, which was important for quantifying the three types of mandibular rotations, was defined as the corpus axis connecting the $\mathrm{Xi}$ and $\mathrm{Pm}$ points according to Rickett's analysis [19]. Scrutinizing Björk's articles, the positions of each implant look obviously parallel to Rickett's corpus axis. The authors concluded the corpus axis (Xi-Pm) could be comparable to Björk's core of the mandible.

Definitions and quantifying methods for the different rotations were adopted from Björk and Skieller's study [2]. Total rotation, meaning the rotation of the mandibular core relative to the cranial base, was set as the angle between the $\mathrm{SN}$ line and corpus axis. The corpus axis that connects the $\mathrm{Xi}$ and $\mathrm{Pm}$ points was set as the mandibular core according to Rickett's analysis [19].

Table 1 Demographic data

\begin{tabular}{lllll}
\hline Number of pairs & & Age & & \\
\cline { 3 - 5 } & & Mean (SD) & Min & Max \\
\hline MZ $(n=36)$ & Male $(n=16)$ & $41.0(7.90)$ & 26 & 57 \\
& Female $(n=20)$ & $38.6(7.56)$ & 24 & 58 \\
DZ $(n=13)$ & Male $(n=7)$ & $42.2(9.15)$ & 34 & 63 \\
& Female $(n=6)$ & $43.67(4.66)$ & 38 & 48 \\
Sibling $(n=26)$ & Male $(n=11)$ & $32(8.32)$ & 20 & 47 \\
& Female $(n=15)$ & $42.8(11.64)$ & 24 & 60 \\
Total $(N=75)$ & & $39(9.26)$ & 20 & 63 \\
\hline
\end{tabular}

Matrix rotation was set as the angle between the SN line and the mandibular plane, and intramatrix rotation as the angle between the mandibular plane and corpus axis respectively. Among the three occlusal planes, the bisected occlusal plane was measured as a line connecting the midpoint of the distobuccal cusps and the overbite midpoint according to Downs [14]. The functional occlusal plane was measured by connecting the midpoint of the upper and lower first molars and the midpoint of the upper and lower premolars according to Wits [15]. The MM bisector plane was measured as the line bisecting the palatal plane and mandibular plane according to Hall-Scott [16] (Fig. 3, Table 2).

\section{Statistical analysis}

All statistical analyses were performed using the SPSS program (IBM SPSS Statistics Version 21) and Microsoft Excel. $P$ values less than 0.01 were considered statistically significant.

Phenotype concordance for the MZ, DZ, and sibling groups was calculated using the intraclass correlation coefficient (ICC), and heritability was calculated by using Falconer's formula [20].

The ICC values for 13 cephalometric parameters were calculated in each group through reliability analysis.

The more similar the value between twins, the less the ICC difference, the higher the ICC value, and the greater the difference between different twins, the higher the ICC.

A higher ICC value indicates higher concordance between variables in the same twin pairs and also indicates greater differences between different twin pairs.

Theoretically, MZ twins share identical genes and DZ twins of the same gender share half of their genes.

Heritability $\left(h^{2}\right)$ indicates genetic factors, and cultural inheritance $\left(C^{2}\right)$ indicates environmental factors that can be calculated as follows using Falconer's formula [21]:

$$
\begin{aligned}
& h^{2}=2\left(\mathrm{ICC}_{\mathrm{MZ}}-\mathrm{ICC}_{\mathrm{DZ}}\right) \\
& \mathrm{C}^{2}=2 \mathrm{ICC}_{\mathrm{DZ}}-\mathrm{ICC}_{\mathrm{MZ}}
\end{aligned}
$$

$\mathrm{ICC}_{\mathrm{MZ}}=$ Intraclass correlation coefficient for MZ twin pairs

$\mathrm{ICC}_{\mathrm{DZ}}=$ Intraclass correlation coefficient for DZ twin pairs

Statistically, heritability means the proportion of trait variance influenced by genetic factors and a high value for heritability means a large effect of genetic factors on any particular phenotype and vice versa. A particular phenotype is the sum of genetic and environmental factors as follows [22]:

Phenotype $(P)=$ Genotype $(G)+$ Environment $(E)$ 


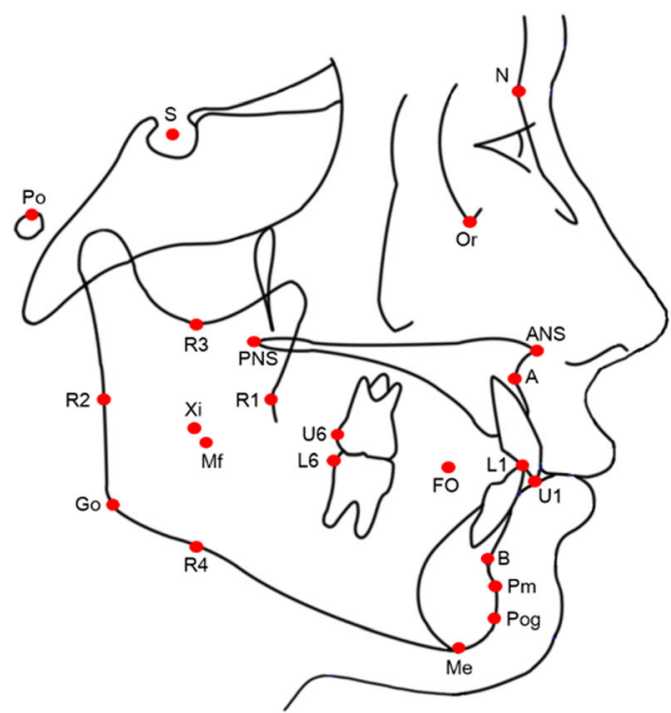

1. sella(S)

2. nasion( $\mathrm{Na})$

3. porion(Po)

4. orbitale(Or)

5. anterior nasal spine(ANS)

6 . posterior nasal spine(PNS)

7. xi point( $\mathrm{Xi})$

8. protuberance menti $(\mathrm{Pm})$

9. mandibular foramen(Mf)

10. Functional occlusal point(FO)

11. upper incisor(U1)

12. lower incisor(L1)

13. upper first molar(U6)

14. lower first molar(L6)

15. $A$ point $(A)$

16. B point(B)

17. pogonion(Pog)

18. gonion(Go)

19. menton(Me)

Fig. 1 Landmarks and reference lines used for the cephalometric analysis

\section{Results}

Statistical analysis indicated that the MZ group showed remarkably higher ICC values for most of the variables compared to the $\mathrm{DZ}$ or sibling groups. This was particularly seen in the ICC for mandibular plane angle to $\mathrm{SN}$, meaning matrix rotation within the $\mathrm{MZ}$ group was 0.87 , which was significantly high. The mean ICC value for 13 cephalometric measurements was $0.85,0.62$, and 0.52 in the MZ, DZ, and sibling groups, respectively (Table 3 ).

Heritability $\left(h^{2}\right)$ and cultural inheritance $\left(C^{2}\right)$ were calculated by inputting the ICC from the MZ, DZ, and sibling groups to Falconer's formula. Heritability and cultural inheritance for 13 variables were calculated using the ICC of the MZ and DZ groups that are displayed in Table 4.

The heritability of the corpus axis to $\mathrm{SN}$ angle (meaning total rotation) was 0.48 when calculated using the MZ/DZ ICC; mandibular plane to $\mathrm{SN}$ angle (meaning matrix rotation) was 0.5 using the MZ/DZ ICC. However, the heritability of the corpus axis to the mandibular plane angle (meaning intramatrix rotation) was 0.14 using the MZ/DZ ICC, which was relatively low (Table 4).

The occlusal plane to the SN line demonstrated higher heritability than to the $\mathrm{FH}$ line in all three occlusal

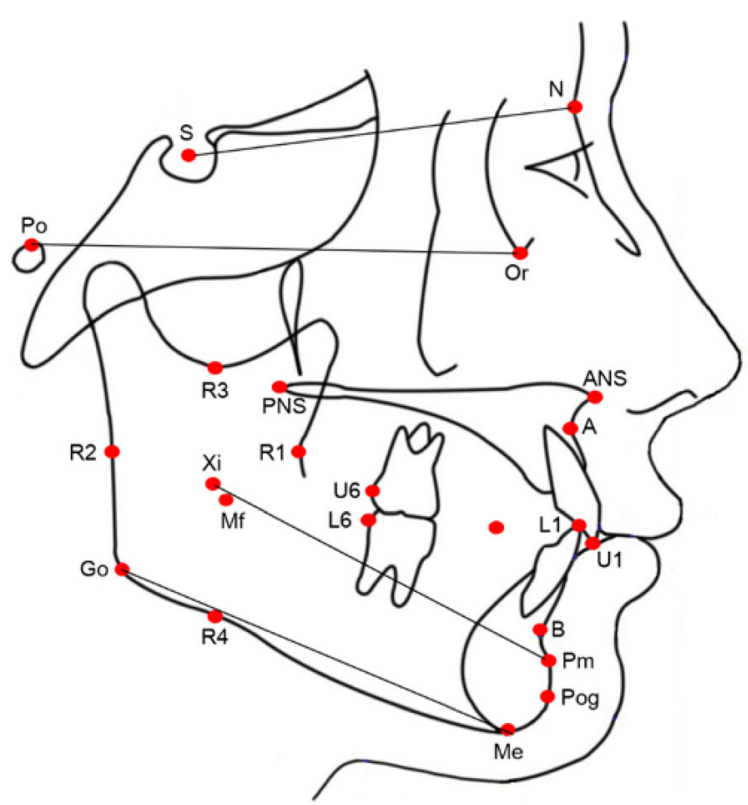

1. SN plane(S-N)

2. FH plane(Po-Or)

3. Mandibular Core (Xi-Pm)

4. Mandibular Plane(Go-Me)

Fig. 2 Three occlusal planes used for the cephalometric analysis 


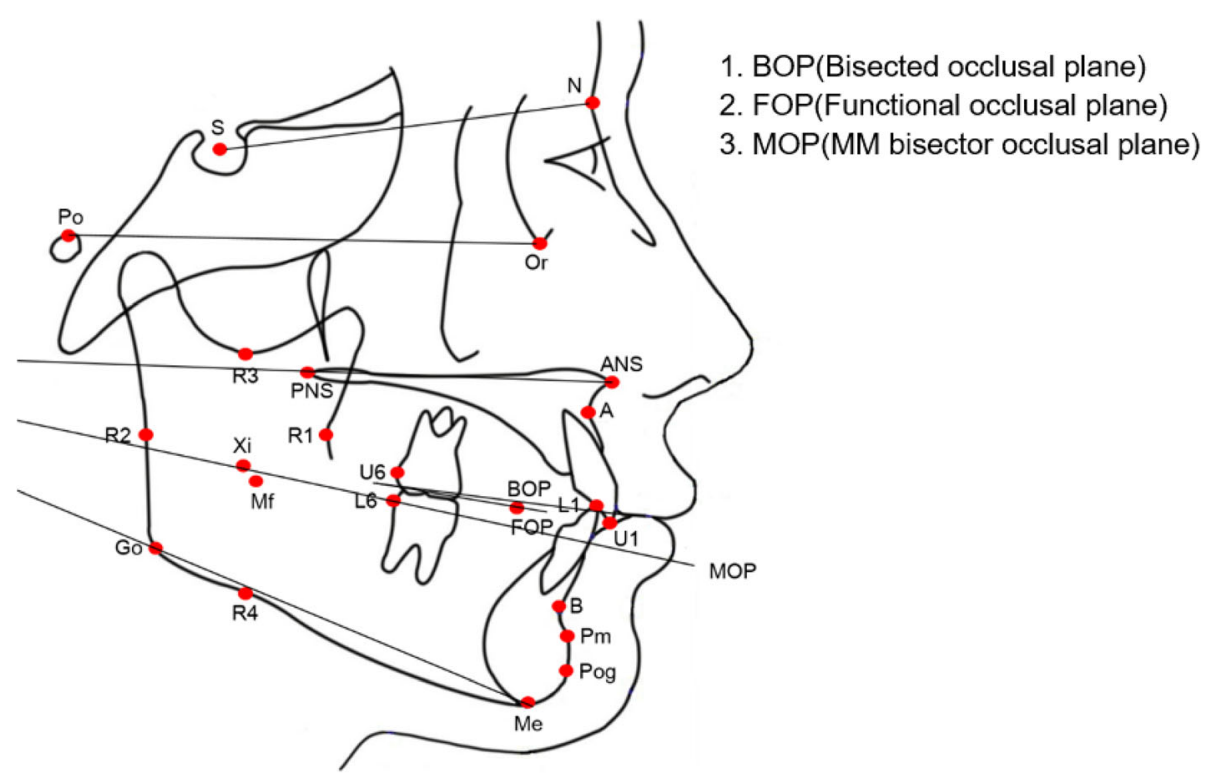

Fig. 3 Plans to evaluate the mandibular rotations

planes. The heritability of the functional occlusal plane to the SN line was 0.52 and to the FH line was 0.76 , respectively. The MM bisector occlusal plane to the $\mathrm{SN}$ line was 0.42 and to the $\mathrm{FH}$ plane was 0.54 respectively, for MZ/DZ, which showed the lowest heritability among the three occlusal planes (Table 4).

\section{Discussion}

Recently, the inheritance characteristics of skeletal, dental, and soft tissue were intensively investigated $[22,23]$. One study was performed that used 13 pairs of MZ and DZ twins and showed similar results as seen in previous studies. The heritability of variables was calculated using Falconer's formula and the results displayed higher heritability for shape than size [23]. These results were also in agreement with Weinberg's study that reported facial shape related to length and breadth of central midfacial structures demonstrated strong heritability [24]. The mandible showed higher heritability than the maxilla [23]. Proportion rather than length itself was more precise for predicting vertical growth in the anterior face. Kim et al also demonstrated that most dental structure variables showed low heritability [23].

Table 2 Three occlusal planes

Bisected occlusal plane (Downs)

- Bisecting line through overlap of the distobuccal cusp of the first

permanent molars and incisors overbite

Functional occlusal plane (Wits)

- The line bisecting the molars and premolars overlaps

MM bisector occlusal plane (Hall-Scott)

- The maxillary-mandibular planes angle bisector
Another study evaluated 30 soft tissue variables using the ICC from 75 pairs of MZ and DZ twins, and their siblings. The results showed stronger heritability in the MZ group compared to the DZ group, and their siblings. The authors also reported that the nasolabial angle and soft tissue chin thickness showed strong heritability [22].

In this study, the heritability of variables related to mandible rotation was specifically investigated. The occlusal plane was further divided into three sections and each section's heritability was calculated (Table 4).

Table 3 Intraclass correlation coefficient (ICC) in MZ, DZ, and sibling groups

\begin{tabular}{llll}
\hline Variables & $\mathbf{I C C}_{\mathbf{M z}}$ & $\mathbf{I C C}_{\mathbf{D Z}}$ & $\mathbf{I C C}_{\text {Sib }}$ \\
\hline Bisected OP (to SN) & $0.83^{* * *}$ & $0.64^{*}$ & $0.62^{* *}$ \\
Bisected OP (to FH) & $0.85^{* * *}$ & 0.35 & 0.48 \\
Functional OP (to SN) & $0.75^{* * *}$ & 0.49 & 0.57 \\
Functional OP (to FH) & $0.79^{* * *}$ & 0.41 & 0.36 \\
MM bisector OP (to SN) & $0.87^{* * *}$ & $0.66^{*}$ & $0.68^{*}$ \\
MM bisector OP (to FH) & $0.86^{* * *}$ & 0.59 & 0.51 \\
Mn. plane angle (to SN): MR & $0.87^{* * *}$ & 0.62 & 0.55 \\
Mn. plane angle (to FH) & $0.86^{* * *}$ & 0.49 & 0.41 \\
Palatal plane angle (to SN) & $0.82^{* * *}$ & 0.48 & $0.65^{*}$ \\
Palatal plane angle (to FH) & $0.82^{* * *}$ & 0.53 & $0.53^{*}$ \\
Corpus axis (to SN): TR & $0.85^{* * *}$ & 0.61 & 0.45 \\
Corpus axis (to FH) & $0.81^{* * *}$ & 0.51 & 0.24 \\
Corpus axis (to MP): IR & $0.74^{* *}$ & $0.67^{*}$ & 0.24
\end{tabular}

MZ Monozygotic twin, DZ Dizygotic twin, Sib Sibling, OP Occlusal plane, SN SN plane, FH FH plane, MP Mandibular plane, MR Matrix rotation, TR Total rotation, $I R$ Intramatrix rotation ${ }^{*} P<.05,{ }^{*} P<.01,{ }^{* * *} P<.001$ 
Table 4 Estimates of heritability $\left(h^{2}\right)$ and cultural inheritance $\left(C^{2}\right)$ between $M Z$ and $D Z$ twins

\begin{tabular}{lll}
\hline Variables & \multicolumn{2}{l}{ MZ and DZ twins } \\
\cline { 2 - 3 } & $\boldsymbol{h}^{\mathbf{2}}$ & $\boldsymbol{C}^{\mathbf{2}}$ \\
\hline Bisected OP (to SN) & 0.38 & 0.45 \\
Bisected OP (to FH) & 1.00 & -0.15 \\
Functional OP (to SN) & 0.52 & 0.23 \\
Functional OP (to FH) & 0.76 & 0.03 \\
MM bisector OP (to SN) & 0.42 & 0.45 \\
MM bisector OP (to FH) & 0.54 & 0.32 \\
Mn. plane angle (to SN): MR & 0.5 & 0.37 \\
Mn. plane angle (to FH) & 0.74 & 0.12 \\
Palatal plane angle (to SN) & 0.68 & 0.14 \\
Palatal plane angle (to FH) & 0.58 & 0.24 \\
Corpus axis (to SN): TR & 0.48 & 0.37 \\
Corpus axis (to FH) & 0.60 & 0.21 \\
Corpus axis (to MP): IR & 0.14 & 0.60
\end{tabular}

MZ Monozygotic twin, DZ Dizygotic twin, Sib Sibling, OP Occlusal plane, SN SN plane, FH FH plane, MP Mandibular plane, MR Matrix rotation, TR Total rotation, IR Intramatrix rotation

${ }^{\mathrm{a}} h^{2}=2\left(\mathrm{ICC} \mathrm{MZ}_{\mathrm{MZ}}-\mathrm{ICC} \mathrm{DZ}\right), \mathrm{C}^{2}=\mathrm{ICC}_{\mathrm{MZ}}-h^{2}$

Our results displayed remarkably high ICC values in the MZ twin group compared to DZ twins or siblings for all variables (Table 3).

However, some results were not significantly different between the two groups. For example, $\mathrm{ICC}_{\mathrm{MZ}}$ and $\mathrm{ICC}_{\mathrm{DZ}}$, for corpus axis to mandibular plane angle, were 0.74 and .067 , respectively, resulting in low heritability. The remarkably low $\mathrm{ICC}_{\text {sib }}$ for corpus axis to mandibular plane angle (0.24) might be due to the small sample size ( $M Z=36$ pairs, $D Z=13$ pairs, siblings 26 pairs). $\mathrm{DZ}$ twin groups that experience the embryonic process in utero together present with higher corpus axis to mandibular plane values compared to siblings, suggesting that intrauterine factors have more effect than postnatal factors.

Unlike the intramatrix rotation, the ICC values for total rotation and matrix rotation were consistent and their heritability was relatively high. Total rotation is the rotation of the mandibular core and is genetically determined to some degree. On the other hand, matrix rotation is a complex process that includes articular growth and changes in mandibular shape caused by remodeling. The heritability for matrix rotation was 0.5 which was higher than that for the intramatrix rotation, 0.14 (Table 4). In this study, the core of the mandible had high heredity compared to the lower border of the mandible (the mandibular plane) relative to the skull base (SN). This means that the lower border of the mandible, having a lot of attached muscles, is determined by function rather than the heredity rate. For example, if the intramatrix rotation was significantly higher than the total rotation in a young long face patient, prediction of the better outcome in growth modification than in patients with a higher value of the total rotation is anticipated. This is because the heredity of intramatrix rotation is lower than of the other two rotations.

The functional occlusal plane showed the highest heritability and the MM bisector occlusal plane the lowest (Table 4). Therefore, the functional occlusal plane is highly inherited and less influenced by the environment; thus, less change during the growth period can be predicted. Although there have been attempts to treat open and deep bite issues by changing the occlusal plane, the stability of treatment results is a constant concern [25]. The results of this study suggest that changing the functional occlusal plane, which has high heritability, can affect treatment results and stability. For example, great change in the occlusal plane to treat open bite issues caused by intruding upper molars might render patients susceptible to relapse because the occlusal plane is highly inherited and less influenced by its environment, including treatment interventions.

The drawback of this study is its small DZ twin sample size compared with the MZ twin or sibling groups (DZ $=13$ pairs, male $=7$ pairs, female $=6$ pairs), which may have caused less consistency in DZ results. Further studies will be necessary with increased twin numbers especially for the DZ twin group.

\section{Conclusion}

The ICC values were remarkably higher for MZ than DZ twins or their siblings for most measurements related to mandibular rotation and the occlusal plane. Total rotation and matrix rotation showed relatively higher heritability compared to intramatrix rotation. Among the three occlusal planes, the functional occlusal plane showed the highest heritability, followed by the bisected occlusal plane and the MM bisector occlusal plane. Therefore, maintaining the occlusal plane and SN to the corpus axis must be considered to establish a stable treatment plan.

\section{Acknowledgements}

Not applicable.

\section{Authors' contributions}

With the submission of this manuscript, I would like to state that: All authors of this research paper have directly participated in the planning, execution, or analysis of this study. All authors of this paper have read and approved the final version submitted. The contents of this manuscript have not been copyrighted or published previously. The contents of this manuscript are not now under consideration for publication elsewhere. The contents of this manuscript will not be copyrighted, submitted, or published elsewhere, while acceptance by the Journal is under consideration. The research is original. There are no directly related manuscripts published or unpublished, by any authors of this paper. There is no conflict of interest to disclose of all authors. The individual role of each author was as follows: Jin Hyeong Kim: collection of data, analysis of data, interpretation of data, and construction of manuscript. Young Ho Kim: conception and design of the article. Soo jin Kim: conception and design of the article. Joohon Sung: conception and design of the article. Yun-Mi Song: conception and design of the article. 
Jeong Won Shin: conception and design of the article. Jae Hyun Park: conception and design of the article. Hwa Sung Chae: conception and design of the article, analysis of data, interpretation of data, and manuscript construction. All the authors read and approved the final manuscript.

\section{Funding}

The authors declare that they have no funding for this research article.

\section{Availability of data and materials}

The data supporting the study can be obtained directly from the authors.

\section{Ethics approval and consent to participate}

This study was approved by the Institutional Review Board of the Ajou University Hospital (IRB No: AJIRB-MED-MDB-18-295). Subjects read and signed informed consent.

\section{Consent for publication}

Not applicable.

\section{Competing interests}

The authors declare that they have no competing interests.

\section{Author details}

${ }^{1}$ Department of Orthodontics, Institute of Oral Health Science, Ajou University School of Medicine, Suwon, South Korea. ${ }^{2}$ Department of Epidemiology, School of Public Health, Seoul National University, Seoul, South Korea. ${ }^{3}$ Department of Family Medicine, Samsung Medical Center, Sungkyunkwan University School of Medicine, Seoul, South Korea. ${ }^{4}$ Postgraduate Orthodontic Program, Arizona School of Dentistry \& Oral Health, A.T. Still University, Mesa, AZ, USA.

Received: 22 May 2020 Accepted: 23 November 2020

Published online: 07 December 2020

\section{References}

1. Björk A. Prediction of mandibular growth rotation. Am J Orthod. 1969;55(6): 585-99.

2. Björk A, Skieller V. Normal and abnormal growth of the mandible. A synthesis of longitudinal cephalometric implant studies over a period of 25 years. Eur J Orthod. 1983;5(1):1-46.

3. Proffit WR, Fields HW Jr, Sarver DM. Contemporary orthodontics. St Louis: Elsevier Health Sciences; 2018.

4. Nakata M, Yu P-I, Davis B, Nance W. The use of genetic data in the prediction of craniofacial dimensions. Am J Orthod Dentofacial Orthop. 1973;63(5):471-80.

5. Savoye I, Loos R, Carels C, Derom C, Vlietinck RJTAO. A genetic study of anteroposterior and vertical facial proportions using model-fitting. Angle Orthod. 1998:68(5):467-70.

6. Johannsdottir B, Thorarinsson F, Thordarson A, Magnusson TE. Heritability of craniofacial characteristics between parents and offspring estimated from lateral cephalograms. Am J Orthod Dentofacial Orthop. 2005;127(2):200-7.

7. Hunter WS. A study of the inheritance of craniofacial characteristics as seen in lateral cephalograms of 72 like-sexed twins. Report of the congress European Orthodontic Society; 1965.

8. Manfredi C, Martina R, Grossi GB, Giuliani M. Heritability of 39 orthodontic cephalometric parameters on MZ, DZ twins and MN-paired singletons. Am J Orthod Dentofacial Orthop. 1997;111(1):44-51.

9. Carels C, Van Cauwenberghe N, Savoye I, Willems G, Loos R, Derom C, et al. A quantitative genetic study of cephalometric variables in twins. Clin Orthod Res. 2001;4(3):130-40.

10. Naini FB, Moss JP. Three-dimensional assessment of the relative contribution of genetics and environment to various facial parameters with the twin method. Am J Orthod Dentofacial Orthop. 2004;126(6):655-65.

11. Amini F, Borzabadi-Farahani A. Heritability of dental and skeletal cephalometric variables in monozygous and dizygous Iranian twins. Orthodontic Waves. 2009:68(2):72-9.

12. Sidlauskas M, Salomskiene L, Andriuskeviciute I, Sidlauskiene M, Labanauskas Z, Vasiliauskas A, et al. Heritability of mandibular cephalometric variables in twins with completed craniofacial growth. Eur J Orthod. 2016;38(5):493-502.

13. Lobb WK. Craniofacial morphology and occlusal variation in monozygous and dizygous twins. Angle Orthod. 1987;57(3):219-33.
14. Downs WBJTAO. Analysis of the dentofacial profile. Angle Orthod. 1956; 26(4):191-212

15. Jacobson A. The "Wits" appraisal of jaw disharmony. Am J Orthod. 1975; 67(2):125-38.

16. Hall-Scott J. The maxillary-mandibular planes angle (MMo) bisector: a new reference plane for anteroposterior measurement of the dental bases. Am J Orthod Dentofacial Orthop. 1994;105(6):583-91.

17. Song Y-M, Lee D-H, Lee MK, Lee K, Lee HJ, Hong EJ, et al. Validity of the zygosity questionnaire and characteristics of zygosity-misdiagnosed twin pairs in the Healthy Twin Study of Korea. Twin Res Hum Genet. 2010;13(3): 223-30.

18. Houston W. The analysis of errors in orthodontic measurements. Am J Orthod. 1983;83(5):382-90.

19. Ricketts RM. A principle of arcial growth of the mandible. Angle Orthod. 1972:42(4):368-86.

20. Falconer DS. Introduction to quantitative genetics. Edinburgh \& London: Oliver ANd Boyd; 1960.

21. Lundström A, McWilliam JS. A comparison of vertical and horizontal cephalometric variables with regard to heritability. Eur J Orthod. 1987;9(2): 104-8.

22. Song J, Chae HS, Shin JW, Sung J, Song YM, Baek SH, et al. Influence of heritability on craniofacial soft tissue characteristics of monozygotic twins, dizygotic twins, and their siblings using Falconer's method and principal components analysis. Korean J Orthod. 2019;49(1):3-11.

23. Kim E, Sung J, Song YM, Chae HS, Mo SS, Kim YH, et al. Heritability of facial skeletal and dental characteristics of monozygotic and dizygotic twins using cephalometric analysis and Falconer's method. J Craniofac Surg. 2018;29(3): e274-e9.

24. Weinberg SM, Parsons TE, Marazita ML, Maher BS. Heritability of face shape in twins: a preliminary study using 3D stereophotogrammetry and geometric morphometrics. Dent 3000. 2013;1(1):14.

25. Park HS, Kim JY, Kwon TG. Occlusal plane change after intrusion of maxillary posterior teeth by microimplants to avoid maxillary surgery with skeletal Class III orthognathic surgery. Am J Orthod Dentofacial Orthop. 2010;138(5): $631-40$

\section{Publisher's Note}

Springer Nature remains neutral with regard to jurisdictional claims in published maps and institutional affiliations.

\section{Submit your manuscript to a SpringerOpen ${ }^{\bullet}$ journal and benefit from:}

- Convenient online submission

- Rigorous peer review

- Open access: articles freely available online

- High visibility within the field

- Retaining the copyright to your article

Submit your next manuscript at $>$ springeropen.com 